\title{
Five-year-old girl with bilateral lower leg pain
}

\author{
C. Kloth $^{1} \cdot$ T. Wowra $^{2} \cdot$ D. Vogele ${ }^{1}$ - M. Beer ${ }^{1}$ - S. A. Schmidt ${ }^{1}$
}

Published online: 1 July 2020

(C) ISS 2020

Keywords Juvenile dermatomyositis · Myopathy

\section{Discussion}

Inflammatory muscular myopathies can be divided into disorders caused by infectious etiologies, e.g., viral or bacterial, or by an autoimmune process (polymyositis, dermatomyositis, and other types) [1]. Juvenile dermatomyositis (JDMS) is a rare form of inflammatory muscular myopathies; however, in children, it is the most frequent subtype of muscular rheumatic disorders with an incidence of 2-7 per million per year [2]. Girls are more often affected than boys [3], and the mean age at diagnosis is 7 years [2]. In addition to bilateral pain of the lower legs and pelvis as in the presented case, skin manifestations with erythema of the eyelids and telangiectasia are typical [4]. Other organ systems besides skin and muscle can be affected, including the gastrointestinal tract and lungs [5]. Involvement of myocardium is rare but also possible.

Besides the typical clinical appearance, an elevation of muscle enzymes in the blood, elektomyographic changes, and a characteristic histopathological result are necessary for diagnosis. Necrosis of muscular fibers, perifascicular atrophy, muscle fiber caliber fluctuations, and perivascular inflammatory in biopsy are pathognomonic. Different imaging modalities are suitable to

The case presentation can be found at https://doi.org/10.1007/ s00256-020-03476-y

C. Kloth and T. Wowra contributed equally to this work.

C. Kloth

christopher.kloth@uniklinik-ulm.de

1 Department for Diagnostic and Interventional Radiology, University Hospital Ulm, Albert-Einstein-Allee 23, 89081 Ulm, Germany

2 Department of Pediatrics and Adolescent Medicine, University Hospital Ulm, Eythstrasse 24, 89075 Ulm, Germany evaluate direct and indirect signs of active myositis. In the context of florid myositis, there is a strong recommendation for MRI of the pelvis and extremities without contrast agent [2]. Diffuse muscular edema and fluid collections can be registered in $\mathrm{T} 2 \mathrm{w}$ imaging but also a contrast enhancement along the affected muscle and fascia. Edema correlates directly with the degree of the disease; however, a normal MRI examination does not exclude the diagnosis of active myositis.

Regularly, an unenhanced MRI of the pelvis and extremities is necessary; however, an injection of contrast agent with enhancement could support the diagnosis. MRI techniques including T2 mapping and spectroscopy have been shown to be suitable to identify the disease and assess the degree of disease activity [6]. Alternatively a strong recommendation for ultrasound exists [7].

The most relevant differential diagnosis is polymyositis; however, JDMS can result in epifascial/perimuscular, perimysial, and subcutaneous edema in addition to intramuscular edema. Especially subcutaneous edema correlates with a poor and chronic prognosis. In this context, the course of the disease can be divided into acute and limited, chronic, and chronic ulcerative disease. Other possible reasons for muscular edema can also be an acute traumatic injury or rhabdomyolysis; however, acute traumatic injury would not give a bilateral symmetric appearance.

Besides diagnosis, MRI may also help to assess the extent and number of lesions in order to guide a biopsy in an area of active disease [1,5].

In the presented case, no biopsy was performed, because of the clinical and imaging-based diagnosis of juvenile dermatomyositis. The complete regression of the symptoms with prednisolone and immunoglobulins supported the diagnosis.

Authors' contributions CK and TW: data collection and manuscript preparation

DV, SAS, and MB: manuscript correction 


\section{Compliance with ethical standards}

Conflict of interest The authors declare that they have no conflicts of interest.

Ethical approval All procedures performed in studies involving human participants were in accordance with the ethical standards of the institutional and/or national research committee and with the 1964 Helsinki declaration and its later amendments or comparable ethical standards.

\section{References}

1. Garcia J. MRI in inflammatory myopathies. Skelet Radiol. 2000 Aug;29(8):425-38.

2. Keller S, Golsari A, Yamamura J. Juvenile Dermatomyositis. Rofo. 2018 Sep;190(9):810-3.

3. Rider LG, Werth VP, Huber AM, Alexanderson H, Rao AP, Ruperto N, et al. Measures of adult and juvenile dermatomyositis, polymyositis, and inclusion body myositis: Physician and Patient/Parent Global Activity, Manual Muscle Testing (MMT), Health Assessment Questionnaire (HAQ)/Childhood Health Assessment Questionnaire (C-HAQ), Childhood Myositis Assessment Scale (CMAS), Myositis Disease Activity Assessment Tool (MDAAT), Disease Activity Score (DAS),
Short Form 36 (SF-36), Child Health Questionnaire (CHQ), physician global damage, Myositis Damage Index (MDI), Quantitative Muscle Testing (QMT), Myositis Functional Index-2 (FI-2), Myositis Activities Profile (MAP), Inclusion Body Myositis Functional Rating Scale (IBMFRS), Cutaneous Dermatomyositis Disease Area and Severity Index (CDASI), Cutaneous Assessment Tool (CAT), Dermatomyositis Skin Severity Index (DSSI), Skindex, and Dermatology Life Quality Index (DLQI). Arthritis Care Res (Hoboken). 2011;63(Suppl 11):S118-57.

4. Shah M, Mamyrova G, Targoff IN, Huber AM, Malley JD, Rice $\mathrm{MM}$, et al. The clinical phenotypes of the juvenile idiopathic inflammatory myopathies. Medicine (Baltimore). 2013 Jan;92(1):25-41.

5. Ladd PE, Emery KH, Salisbury SR, Laor T, Lovell DJ, Bove KE. Juvenile dermatomyositis: correlation of MRI at presentation with clinical outcome. AJR Am J Roentgenol. 2011 Jul;197(1):W153-8.

6. Maillard SM, Jones R, Owens C, Pilkington C, Woo P, Wedderburn LR, et al. Quantitative assessment of MRI T2 relaxation time of thigh muscles in juvenile dermatomyositis. Rheumatology (Oxford). 2004 May;43(5):603-8.

7. Leeuwenberg KE, Albayda J. Muscle ultrasound in inflammatory myopathies: a critical review. J Rheum Dis Treat. 2019;069. https://doi.org/10.23937/2469-5726/1510069.

Publisher's note Springer Nature remains neutral with regard to jurisdictional claims in published maps and institutional affiliations. 